\title{
High-spatial Resolution Compositionally-sensitive Imaging of Metallic Particles using Plasmon Energy-loss Electrons in TEM
}

\author{
EDEN M. HUNT, ${ }^{*}$ ZHONG LIN WANG, ${ }^{*}$ NEAL D. EVANS $\dagger$ and JANET M. HAMPIKIAN*‡ \\ ${ }^{*}$ School of Materials Science and Engineering, Georgia Institute of Technology, Atlanta, GA 30332-0245, U.S.A. \\ †Oak Ridge Institute for Science and Education, P.O. Box 117, Oak Ridge, TN 37831-0117, U.S.A.
}

\begin{abstract}
High spatial resolution elemental maps have been demonstrated in studies of $\mathrm{Y}^{+}$implanted alumina and $\mathrm{Al} / \mathrm{Ti}$ multilayer specimens using energy-filtered transmission electron microscopy. A spatial resolution of $\sim 2 \mathrm{~nm}$ is achieved. Aluminum containing particles formed in $\mathrm{Y}^{+}$implanted alumina have been identified using energy-filtered images formed with plasmon energy-loss electrons. Noncrystalline aluminum containing particles in specimens implanted at lower ion energies are also observed and the image contrast can be reasonably interpreted using a compositional shell model. Published by Elsevier Science Ltd
\end{abstract}

Key words: energy-filtering, aluminum, alumina, plasmon, compositionally sensitive imaging, spatial resolution.

\section{INTRODUCTION}

Many materials problems are closely related to the compositions of the phases present in the system. Although crystal lattices can be determined reasonably well using high-resolution electron microscopy, determination of local chemistry, particularly at high spatial-resolution, remains a rigorous challenge. Quantitative compositional imaging is vitally important for characterizing advanced materials. Most chemical imaging is performed using scanning electron microscopy (SEM) and scanning transmission electron microscopy (STEM). In STEM, the electron beam from a field emission gun is focused onto the surface of the specimen using a condenser lens, and the beam is scanned over the specimen with the use of deflection coils. Characteristic inelastic scattering signals emitted from the interaction volume of the electron probe with the specimen are determined by the thickness-projected elemental concentrations, provided there is no beam broadening or fluctuation in incident beam current. The detected inelastic scattering signals at each beam scanning position are displayed on a phosphor screen producing a contrast pattern which is an approximate representation of the chemical positions in the specimen. The interaction of an incident electron with the specimen also causes the emission of X-rays, Auger electrons and secondary electrons. X-rays and Auger signals are used extensively to obtain chemical information from the volume and the surface of a specimen, respectively, and the secondary electron signal allows surface morphology to be imaged in both SEM and STEM.

†Corresponding author.
The energy-filtering system developed in recent years has made it possible to perform chemically sensitive imaging in a transmission electron microscope (TEM) (Reimer, 1995). Characteristic inelastic signals observed in electron energy-loss spectra provide fingerprints of the elements present in the specimen. By separating the electrons with different energy-losses at the exit face of an electron energy-loss spectrometer (EELS), and introducing an energy-selecting system that allows only the electrons with a specific energy-loss to pass through the imaging system, and finally re-dispersing the electrons with a set of magnetic lenses in correspondence to the real space distribution, compositionally sensitive images may be obtained (Reimer et al., 1990; Shuman et al., 1986; Krivanek et al., 1995; Craven and Colliex, 1977). Compositionally sensitive imaging, in general, relies on the signal of the inner shell ionization edge, the intensity of which is largely affected by the threshold energy-loss and the ionization cross-section of the edge. Therefore, the spatial resolution of the image is strongly affected by the signal-to-noise ratio (Wang and Shapiro, 1995). In this respect, lower loss electrons from the plasmon or valence region of the energy loss spectrum $(10-100 \mathrm{eV})$ are favourable for this type of imaging due to the much higher signal intensity, provided any delocalization effects are small in comparison to the required spatial resolution.

In this paper, compositionally sensitive imaging using plasmon energy-loss electrons is explored for a special case in which nanoscale particles are present in an amorphous substrate, and for an atomically sharp Al/Ti interface. It will be shown that the energy filtering system is uniquely capable of imaging and identifying the nature of the particles. A spatial resolution better than $2 \mathrm{~nm}$ can be achieved. 


\section{EXPERIMENTAL METHODS}

Single crystal alumina $(-1-1 \overline{2} 3)$ was ion implanted at ambient temperature with either 100 or $170 \mathrm{keV}$ singly charged yttrium ions to a fluence of $5 \times 10^{16}$ ions $/ \mathrm{cm}^{2}$. Planar view specimens were prepared for TEM observations using standard dimple grinding and ion beam milling techniques. Energy filtered transmission electron microscopy experiments were performed with a Gatan Imaging Filter $\left(\mathrm{GIF}^{(\times)}\right)$interfaced to a Philips CM30 TEM operated at $300 \mathrm{kV}$. The low-loss images were gain normalized, $512 \times 512$ pixels in size and were recorded with an exposure time of $1 \mathrm{~s}$ and an energy-selecting window of $5 \mathrm{eV}$. Images acquired using core-loss electrons were recorded with an exposure time of $30 \mathrm{~s}$ and an energy-selecting window of $30 \mathrm{eV}$. Additional information was obtained by electron diffraction, bright field imaging and energy dispersive X-ray spectroscopy with an oxygen sensitive detector in a Hitachi HF-2000 TEM operated at $200 \mathrm{kV}$.

$\mathrm{Al} / \mathrm{Ti}$ polycrystalline multilayer specimens were prepared by sputtering as described in detail elsewhere (Shechtman et al., 1994). Cross-sectional TEM specimens were prepared which provided atomically sharp, epitaxial interfaces. The $\mathrm{Al} / \mathrm{Ti}$ specimen was imaged using a JEOL 3010 TEM at $300 \mathrm{kV}$ equipped with a GIF system. Some of the EELS spectra were acquired using a Hitachi HF-2000 TEM operated at $200 \mathrm{kV}$.

\section{COMPOSITIONALLY SENSITIVE IMAGING}

The first specimen examined was single crystal alumina implanted at ambient temperature with $100 \mathrm{keV}$ $\mathrm{Y}^{+}$to a fluence of $5 \times 10^{16}$ ions $/ \mathrm{cm}^{2}$. Ion channelling, Knoop microhardness measurements, and transmission electron microscopy (TEM) all indicate that the alumina surface layer was amorphized by the implantation (Hunt et al., 1996a, 1996b; Hunt and Hampikian, 1997). Bright field TEM revealed a lack of diffraction contrast in this sample and displayed only a diffuse electron diffraction haze associated with the presence of an amorphous phase. However, an optical absorption feature in the near ultraviolet indicated the presence of nanostructures in this sample. For this reason energy-filtered imaging was carried out in order to image these nanostructures (Evans et al., 1995). Before presenting the energyfiltered images an understanding of the valence/plasmon electron energy loss patterns of the elements involved is instructive. Single-scattering energy-loss spectra from standard specimens (Ahn and Krivanek, 1983) of alumina $\left(\mathrm{Al}_{2} \mathrm{O}_{3}\right)$, aluminum $(\mathrm{Al})$ and yttria $\left(\mathrm{Y}_{2} \mathrm{O}_{3}\right)$ are shown in Fig. 1; these are all possible components of this system. The strong peak at about $38 \mathrm{eV}$ is the $\mathrm{Y}-\mathrm{N}_{2,3}$ ionization edge. Both the $\mathrm{Al}$ and $\mathrm{Y}_{2} \mathrm{O}_{3}$ spectra show a loss feature at $15 \mathrm{eV}$ and the $\mathrm{Al}_{2} \mathrm{O}_{3}$ spectrum has a broad feature centered at approximately $24 \mathrm{eV}$. A typical energy loss spectrum from one of the yttrium implanted alumina samples is also shown in Fig. 1. This spectrum, which is offset along the $y$-axis for ease of

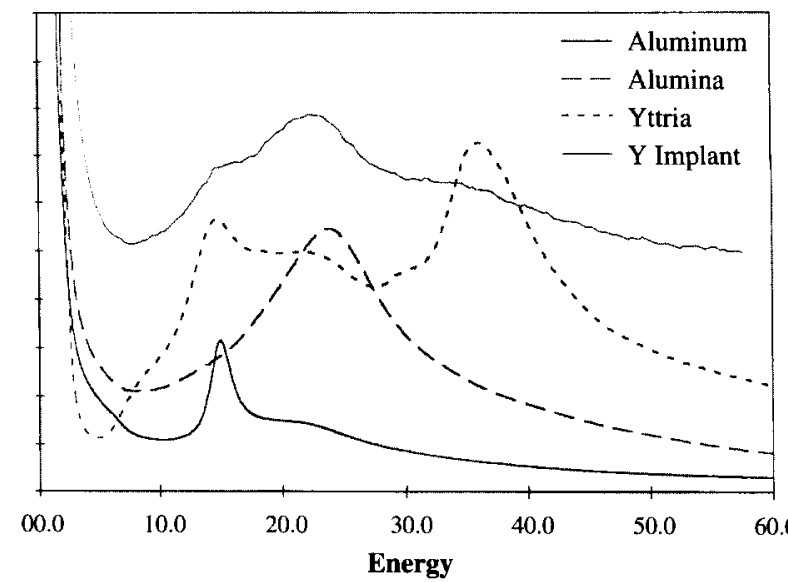

Fig. 1. A comparison of EELS spectra acquired from an aluminum foil, alumina, yttria and a yttrium implanted alumina sample for selecting the characteristic peaks which might be used for forming energy-filtered images. The implanted sample spectrum has been offset along the $y$-axis for clarity.

viewing, contains both the broad $\mathrm{Al}_{2} \mathrm{O}_{3}$ feature at $24 \mathrm{eV}$ and the sharper peak at $15 \mathrm{eV}$. This comparison of spectra indicates that energy-filtered images formed using the electrons with energy-losses of $15 \mathrm{eV}, 25 \mathrm{eV}$ and $40 \mathrm{eV}$ may yield information about the chemical nature of the nanostructures formed during this ion implantation.

A zero-loss electron image of an implanted area is shown in Fig. 2(a). In this image the particles present in the sample are not apparent and the gradual contrast variation is due to the increasing thickness of the sample. Imaging with $15 \mathrm{eV}$ energy-loss electrons (Fig. 2(b)), using a $5 \mathrm{eV}$ energy window, revealed particles within in the alumina matrix. These particles have sizes which range from $20-100 \mathrm{~nm}$. A striking phenomenon is the fine $4-10 \mathrm{~nm}$ substructure within the particles, possibly indicating compositional gradients within the particles. In the image formed by the $25 \mathrm{eV}$ energy-loss electrons (Fig. 2(c)), the bright features (particles) observed in Fig. 2(b) are darker than the surrounding matrix. This result indicates that these particles are not composed of alumina. In addition, the image recorded using the $40 \mathrm{eV}$ energy-loss electrons (Fig. 2(d)) also shows the particles with a lower intensity than the surrounding matrix material. As discussed above, the $38 \mathrm{eV}$ edge is only present in the spectrum of $\mathrm{Y}_{2} \mathrm{O}_{3}$, thus, this image confirms that the particles are not yttria. All of these images support the conclusion that these particles are neither alumina nor yttria.

Similar investigations on another specimen which was implanted with $170 \mathrm{keV} \mathrm{Y^{+ }}$ to a fluence of $5 \times 10^{16}$ ions $/ \mathrm{cm}^{2}$ resulted in comparable images. The zero-loss filtered image (Fig. 3(a)) shows very weak contrast from some of the crystalline particles contained in this sample. The $25 \mathrm{eV}$ loss image (Fig. 3(c)) demonstrates that these particles are not alumina, while the image recorded from the $15 \mathrm{eV}$ energy-loss electrons (Fig. 3(b)) shows many more particles than are apparent in the other images. This is in agreement with the observations shown in 

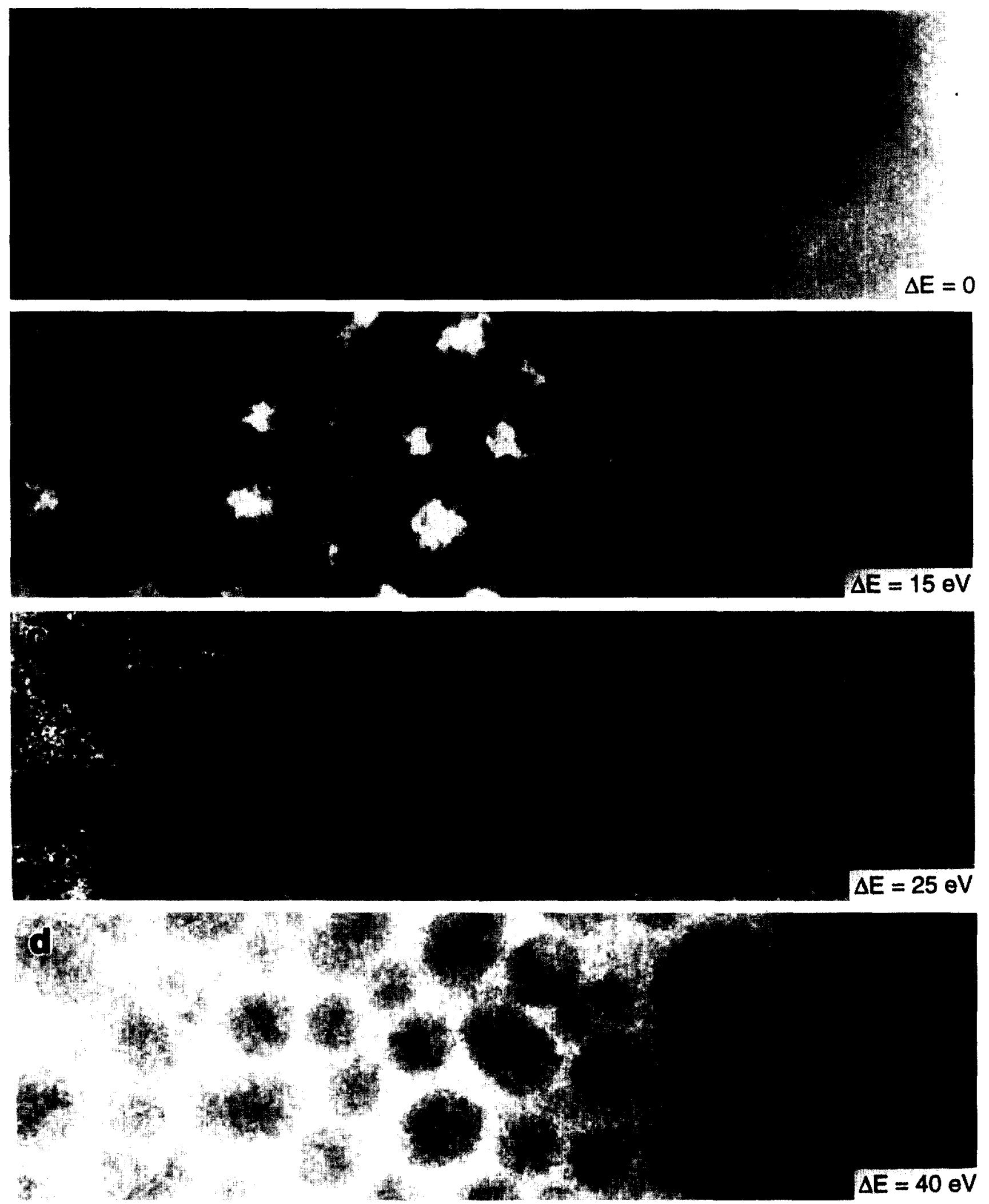

Fig. 2. (a) Zero-loss, (b) $15 \mathrm{eV}$ plasmon-loss, (c) $25 \mathrm{eV}$ plasmon-loss and (d) $40 \mathrm{eV}$ peak energy-selected TEM images from the same specimen region of a $100 \mathrm{keV} \mathrm{Y}^{+}$implanted alumina specimen. The energy selection window was centered at these energies specified and its width was $5 \mathrm{eV}$.

Fig. 2, with the exception that these particles demonstrate little or no substructure. The particle sizes are in the range of $10-15 \mathrm{~nm}$, much smaller than those shown in Fig. 2, despite being the result of the implantation of an identical amount of yttrium $\left(5 \times 10^{16}\right.$ ions $\left./ \mathrm{cm}^{2}\right)$. An oxygen 'jump-ratio' image (post $\mathrm{O}-\mathrm{K}$ edge divided by pre $\mathrm{O}-\mathrm{K}$ edge) from the same area (Fig. 3(d)) shows that the particles are deficient in oxygen with respect to the surrounding matrix material. This result also agrees with previous energy dispersive $\mathrm{X}$-ray spectroscopy (EDS) 

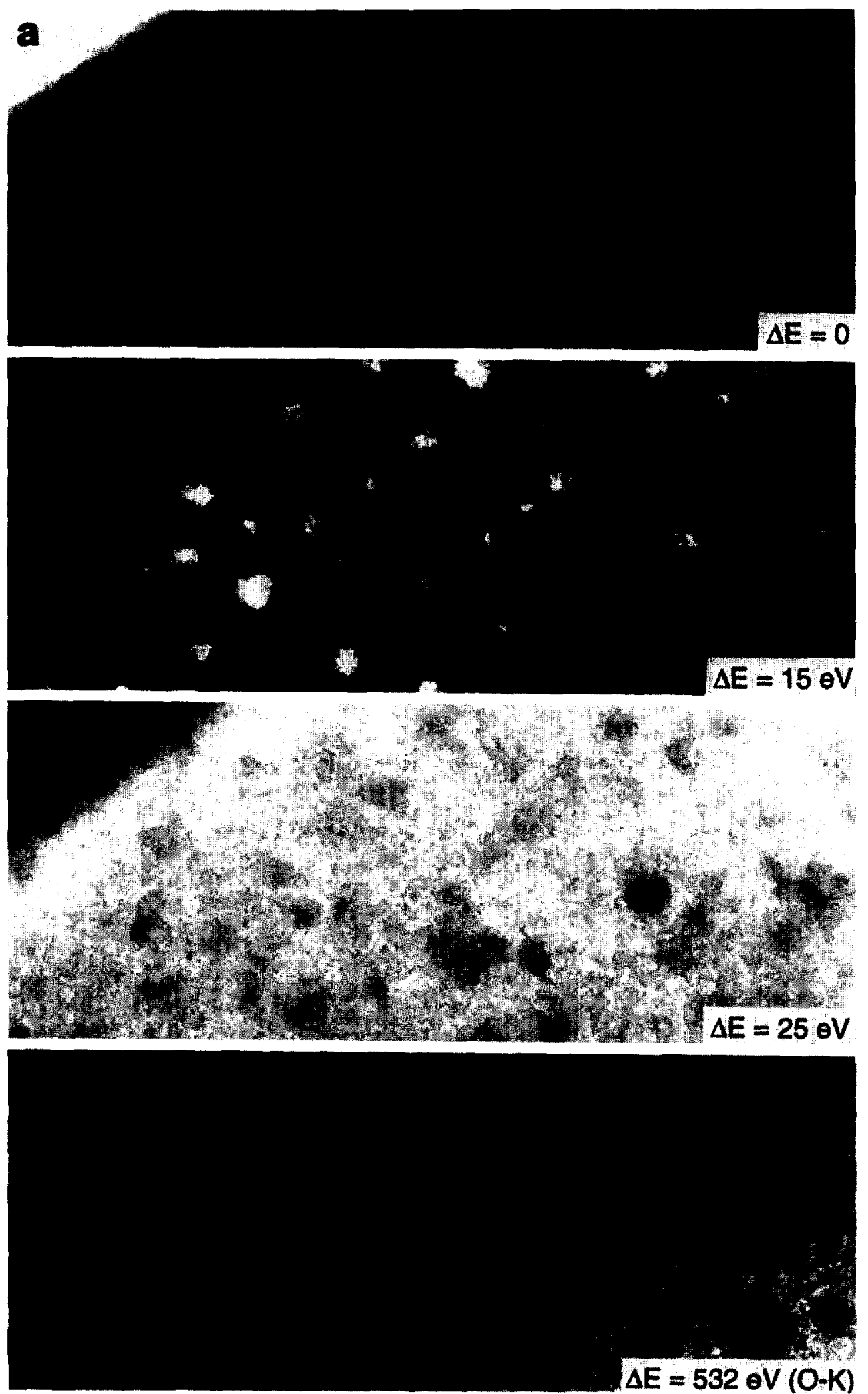

Fig. 3. (a) Zero-loss, (b) $15 \mathrm{eV}$ plasmon-loss, (c) $25 \mathrm{eV}$ plasmon-loss and (d) oxygen jump ratio energy-selected TEM images from the same specimen region of a $170 \mathrm{keV} \mathrm{\textrm {Y } ^ { + }}$ implanted alumina specimen. The energy selection window was centered at these energies specified and its width was $5 \mathrm{eV}$.

analysis which showed that the particles are oxygen deficient with respect to the matrix material (Hunt et al. 1996a, 1996b; Hunt and Hampikian, 1997).

To finalize our identification of the nature of the observed particles, EELS spectra were acquired using electrons transmitted through the particles, the matrix and a standard thin film specimen of Al with surface oxidation, as shown in Fig. 4(a). It is apparent that the $15 \mathrm{eV}$ peak observed in the spectrum from the particle bearing region agrees well with the volume plasmon peak of metallic Al, and there is no detectable metallic $\mathrm{Al}$ in the matrix. The $25 \mathrm{eV}$ peak observed in the 

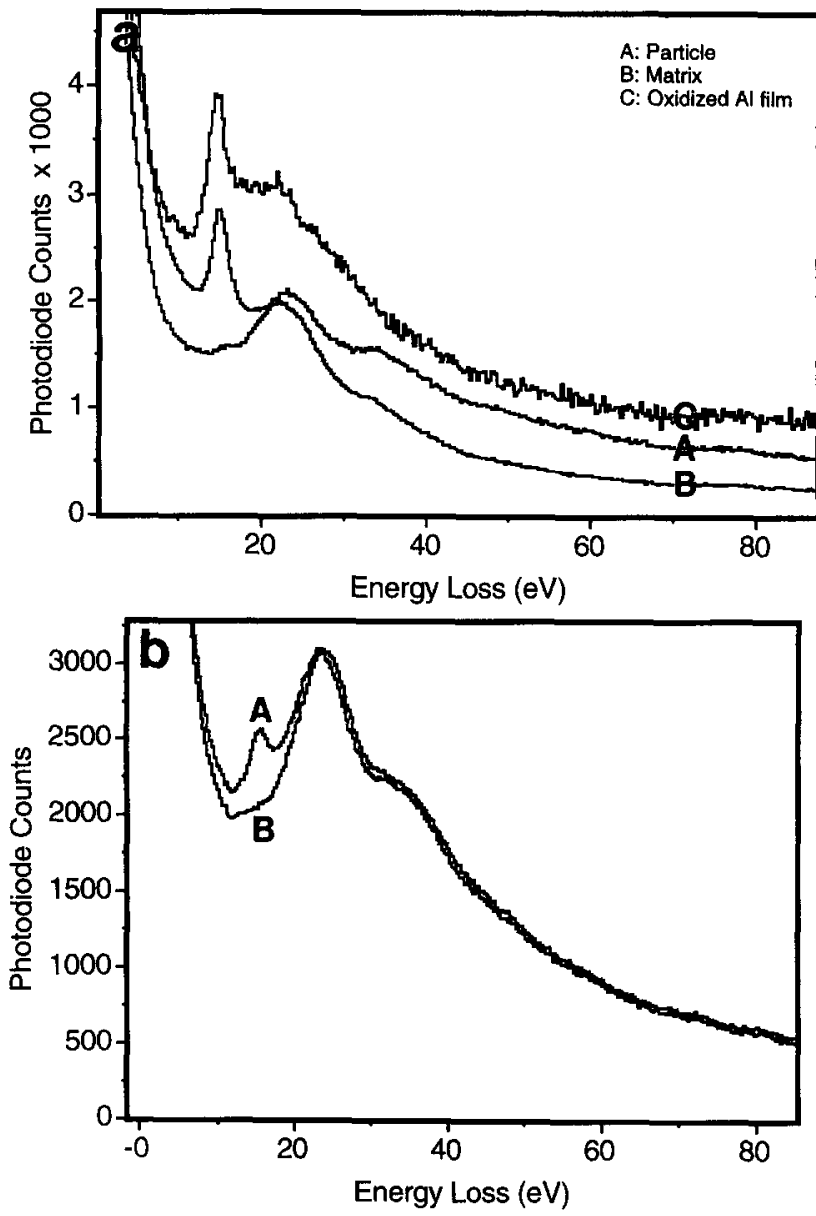

Fig. 4. Energy-loss spectra from (A) an embedded particle and (B) the adjacent amorphous matrix for (a) a large particle and

(b) a small particle, where the spectrum $\mathrm{C}$ was acquired from a standard thin $\mathrm{Al}$ foil covered by alumina oxidation layers.

standard Al specimen is due to the volume excitation of the unavoidable alumina oxidation layer at the top and bottom surfaces of the metal film. The spectra from the embedded particle and the oxidized Al standard are quite similar in major characteristics, allowing the conclusion that the observed particles are metallic Al. Fig. 4(b) shows a comparison of two spectra acquired from a small size $(\sim 5 \mathrm{~nm})$ particle and its adjacent matrix. The intensity of the $15 \mathrm{eV}$ peak in the particle's spectrum is significantly reduced due to the smaller particle size which results in a decrease in the amount of thickness-projected metallic $\mathrm{Al}$ in the beam illumination region.

The particles formed in the alumina matrix implanted with $170 \mathrm{keV} \mathrm{Y}^{+}$clearly show solid contrast with no substructure. In addition, these particles are crystalline. Electron diffraction patterns recorded from a large area have been used to determine the lattice constant of the particle material, with the aid of a standard $\mathrm{Au}$ diffraction pattern recorded under the same imaging conditions. The particles have an FCC structure with a lattice parameter of $a=0.411 \pm 0.002 \mathrm{~nm}$ (Fig. 5(a)). High resolution lattice images of the particles were used to carry out an independent calculation of the lattice parameter (Fig. 5(b)). The particle in Fig. 5 is oriented along [011], and the lattice parameter $a=0.41 \mathrm{~nm} \pm 0.03 \mathrm{~nm}$, is in good agreement with the diffraction measurements. Therefore, the similarity between the particle crystallography and that of pure aluminum (which is FCC with a lattice parameter of $0.404 \mathrm{~nm}$ ), in conjunction with the EFTEM results suggests that the particles are metallic aluminum nanocrystals with a slightly dilated lattice parameter, possibly due to the incorporation of a small amount of yttrium.

\section{SPATIAL RESOLUTION}

As observed in Fig. 2, the Al-rich particles show contrast suggestive of compositional gradients present in the particles. To estimate the optimum spatial resolution that can be achieved using plasmon energy-loss electrons, image processing is carried out. This processing consists of subtracting the contribution of the alumina matrix from the $15 \mathrm{eV}$-loss image. This contribution was estimated using adjacent loss images and subtracted from the original $15 \mathrm{eV}$-loss image (Evans et al., 1995). Fig. 6(a) shows a processed $15 \mathrm{eV}$ energy-filtered image from the specimen implanted with $100 \mathrm{keV} \mathrm{Y}^{+}$. A line scan across a particle (indicated by a line in Fig. 6(a)) is shown in Fig. 6(b). This line scan contains two peaks representing the intensity of the particle image along the indicated line. The full width at half maximum of this intensity is measured to be $W=3.7 \pm 0.3 \mathrm{~nm}$. This value includes not only the finite width of the Al wall but also the contrast blurring due to the delocalization effect in plasmon excitation.

In this implanted sample both the particles and the surrounding matrix are amorphous, therefore it is difficult to use conventional imaging or diffraction techniques to estimate the local thickness of the $\mathrm{Al}$ substructure present in these particles. Therefore, another specimen with a known interface structure was used to determine the spatial resolution that can be expected using $15 \mathrm{eV}$ loss electrons. In practice, it is impossible to preserve a metallic Al surface without oxidation in conventional TEM with a vacuum of $10^{-7}-10^{-8}$ Torr in the column. The interface studies were carried out using a cross-sectional Ti/Al multilayer sample. Fig. 7(a) and (b) show a pair of high-resolution lattice images of an $\mathrm{Al} / \mathrm{Ti}$ (111) interface recorded using zero-loss and $\mathrm{Al}$ plasmon-loss electrons, respectively. The $\mathrm{Al} / \mathrm{Ti}$ interface is atomically sharp and is oriented parallel to the incident beam direction. It is apparent that the crystal lattice fringes are resolved in the image recorded using the inelastically scattered electrons. The overall contrast of the plasmon-loss energy-selected image may provide compositionally sensitive information, but the lattice fringes are still governed by phase contrast (Wang, 1997), as in conventional HRTEM. Therefore, the bright fringes do not necessarily represent the $\mathrm{Al}$ (or $\mathrm{Ti}$ ) atomic planes.

To estimate the spatial resolution of the plasmon-loss electron image, a line scan is made across the Al/Ti interface, and the result is shown in Fig. 7(c). The fine oscillation in the intensity profile is due to the lattice fringes. The intensity profile drops within a distance of 

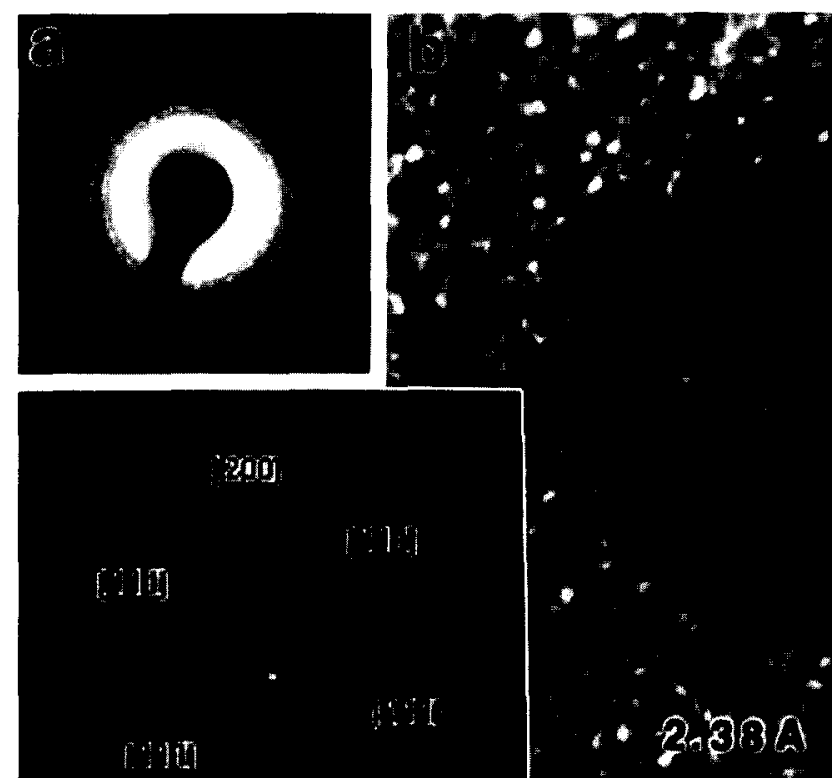

1.20ij

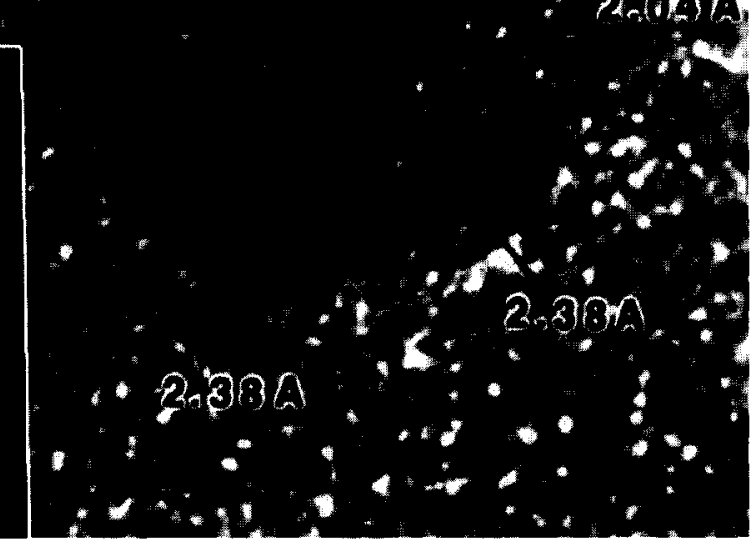

Fig. 5. (a) A selected area electron diffraction pattern and (b) a high-resolution TEM image of a single solid particle from a $170 \mathrm{keV}$ $\mathrm{Y}^{+}$implanted alumina specimen.

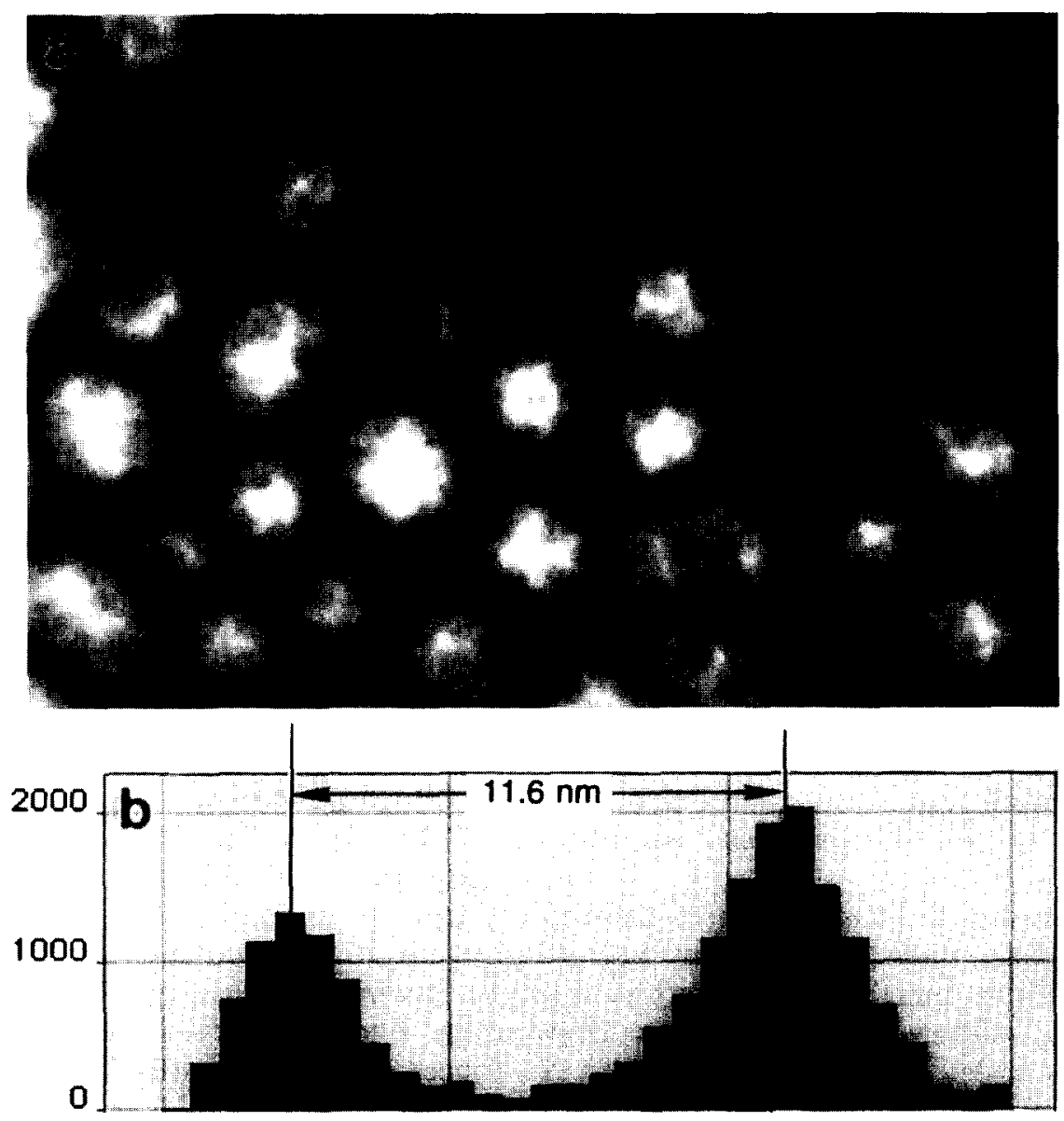

Fig. 6. (a) A background subtracted $15 \mathrm{eV}$ plasmon-loss energy-filtered TEM image showing only the contribution of the metallic $\mathrm{Al}$ (b) A line scan across particle A (indicated in (a)) for the estimation of the thickness of the Al wall.

$3 \mathrm{~nm}$ around the interface. Thus, the spatial resolution is approximately $r_{\mathrm{i}}=1.5$ to $1.8 \mathrm{~nm}$. This spatial resolution is determined by the non-localized scattering of valence electrons (Wang and Shapiro, 1995). Using this value, the thickness of the $\mathrm{Al}$ structure in the particles shown in Fig. 6(b) is estimated to be $\Delta d \approx W-r_{\mathrm{i}} \approx 2.1 \pm 0.4 \mathrm{~nm}$. 

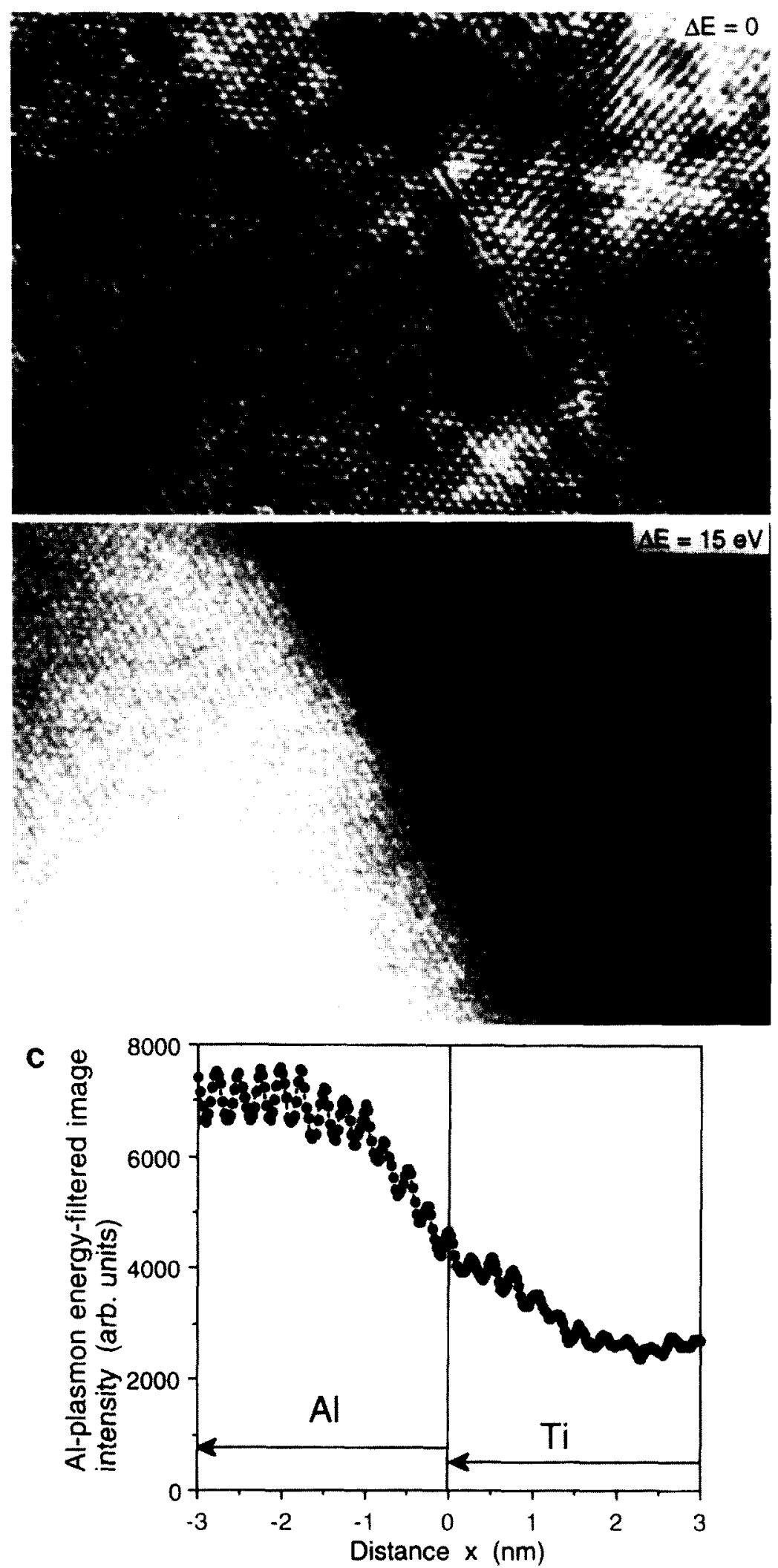

Fig. 7. (a) Zero-loss and (b) $15 \mathrm{eV}$ Al-plasmon loss energy-selected HREM images of an edge-on Al/Ti (111) interface. Energy

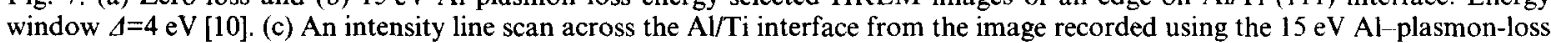
electron image. This curve determines the spatial resolution of valence-loss electron imaging, provided the interface is atomically sharp and there is no interdiffusion. 
a
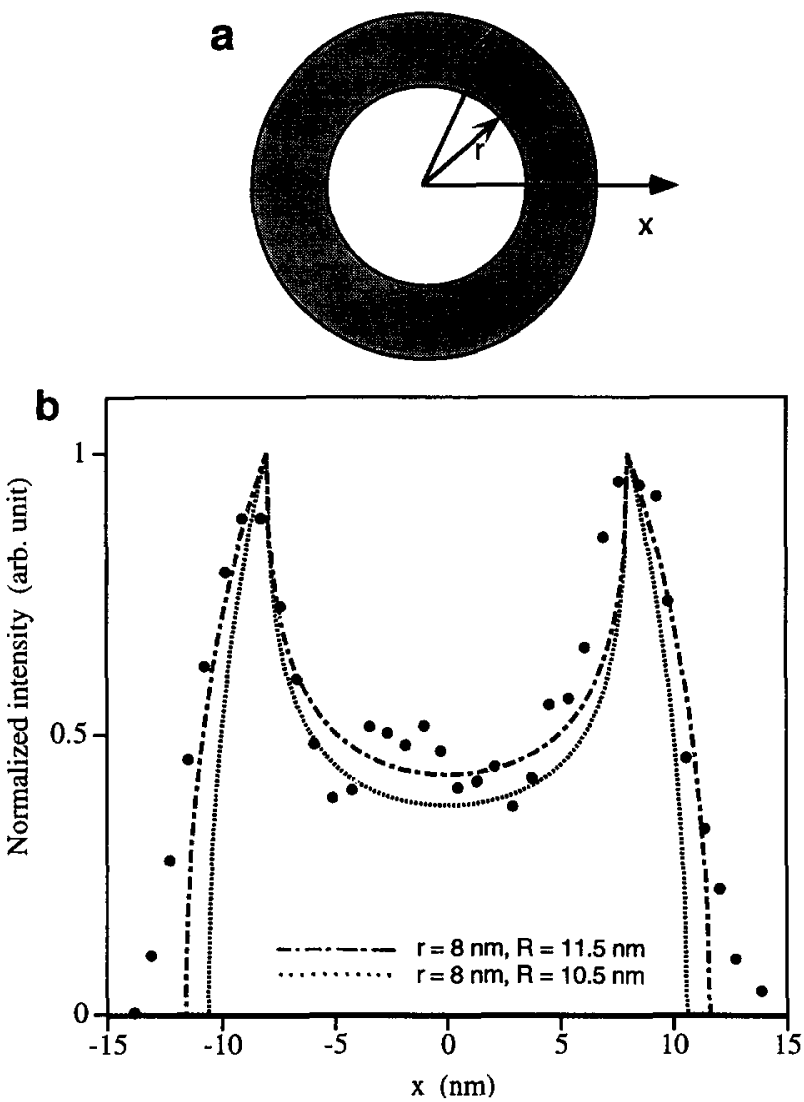

Fig. 8. (a) A spherical shell model of a compositionally porous Al particle, and (b) the projected atom density of the shell particle along the beam direction for two cases in comparison with the experimentally observed profile obtained from the particle B in Fig. 6(a).

\section{DISCUSSION}

\section{Thickness projected Al profile across a shell particle}

The formation of metallic particles in an alumina matrix is not surprising. This has been observed previously in electron beam induced reduction of alumina in STEM and TEM (Humphreys et al., 1985; Wang, 1991). The reduction mechanism in that study was determined to be an internal Auger decay process for the electron stimulated surface desorption (Feibelman and Knotek, 1978). However, in this study, the incident beam is $\mathrm{Y}^{+}$rather than electrons. The mechanism for this $\mathrm{Al}$ particle formation is under investigation.

As shown previously in Figs 2 and 6, the $\mathrm{Al}$ in the particles shows a sort of porosity or compositional substructure, and some particles, as indicated with A and B in Fig. 6(a), show a single shell shape. If metallic $\mathrm{Al}$ is distributed in a shell, as defined by an inner and outer radii (Fig. 8(a)), the projected atom density can be calculated across the particle for various radii (dotted and dashed lines in Fig. 8(b)). The two calculated curves are normalized at $x=r$ for comparing the change in line scan profiles. An experimentally observed line scan intensity is also plotted. It appears that the best fit is obtained for the case with $r=8 \mathrm{~nm}$ and $R=11.5 \mathrm{~nm}$. On the other hand, with consideration of the broadening of the profile by the finite resolution of the plasmon energy-loss electron, a $1 \mathrm{~nm}$ width should be subtracted approximately from the outer radius. A calculation for $r=8 \mathrm{~nm}$ and $R=10.5 \mathrm{~nm}$ is given in Fig. 8(b). Therefore, the thickness of the shell at the intersection of the line scan is $\Delta d=R-r=2.5 \mathrm{~nm}$, which is in good agreement with the estimation given above $(2.1 \pm 0.4 \mathrm{~nm})$.

\section{Surfacelinterface plasmon}

Since our experiments were performed by choosing a $5 \mathrm{eV}$ width energy window centered at $15 \mathrm{eV}$, one might wonder about the contribution from the interface plasmon peak. If we assume the particle is sufficiently large, the energy of the Al-alumina interface plasmon (Wang, 1996) is located at

$$
\omega_{\mathrm{i}}=\frac{\omega_{\mathrm{p}}}{\sqrt{\varepsilon+1}}
$$

where the dielectric constant $\varepsilon$ of alumina is approximately 9 , and the volume plasmon energy $h \omega_{\mathrm{p}}$ of $\mathrm{Al}$ is $15 \mathrm{eV}$. Thus, the interface plasmon energy is $h \omega_{\mathrm{i}} \approx 4.7 \mathrm{eV}$, falling well outside of the energy-selection window. Therefore, the energy-filtered images are formed only by the volume plasmon excitation.

\section{CONCLUSIONS}

In this paper, high spatial resolution, chemically sensitive images have been demonstrated in studies of $\mathrm{Y}^{+}$implanted alumina and $\mathrm{Al} / \mathrm{Ti}$ multilayer specimens using transmission electron microscopy with an electron energy-filtering system. Aluminum particles formed in $\mathrm{Y}^{+}$implanted alumina have been identified using the energy-filtered electron images of plasmon energy-losses and electron energy-loss spectra. A spatial resolution of $\sim 2 \mathrm{~nm}$ is achieved. Crystalline Al particles are formed in alumina implanted at $170 \mathrm{keV}$, while larger compositionally porous amorphous $\mathrm{Al}$ particles are formed in specimens implanted at $100 \mathrm{keV}$. The plasmon energy-loss electron image of the porous Al particles can be reasonably interpreted using a compositional shell model.

Acknowledgements-This material is based upon the work supported by the National Science Foundation under grant No. DMR-9624927, by the Division of Materials Sciences, U.S. Department of Energy, under contract DE-AC05-96OR22464 with Lockheed Martin Energy Research Corporation, through the SHaRE Program under the contract DE-AC05-76OR00033 with Oak Ridge Associated Universities, and by the Office of Naval Research through the Molecular Design Institute at Georgia Institute of Technology.

\section{REFERENCES}

Ahn, C. C. and Krivanek, O. L., 1983. EELS Atlas. Gatan Inc., Warrendale, PA; and Arizona State University.

Craven, A. J. and Colliex, C., 1977. High resolution energy filtered images in STEM. I. Microsc. Spectrosc. Electron., 2, 511-522. 
Evans, N. D., Bentley, J. and Zinkle, S. J., 1995. Energy-filtered plasmon images of $\mathrm{MgAl}_{2} \mathrm{O}_{4}$ implanted with $\mathrm{Al}^{+}$and $\mathrm{Mg}^{+}$ions. In Proc. Microscopy and Microanalysis, pp. 266-267.

Feibelman, P. J. and Knotek, M. L., 1978. Reinterpretation of electron-stimulated desorption data from chemisorption systems. Phys. Rev. B, 18, 6531-6539.

Humphreys, C. J., Salisbury, I. G., Berger, S. D., Timsit, R. S. and Mochel. M. E., 1985. Nanometre-scale electron beam lithography. In Inst. Phys. Conf. Ser. No. 78, Ch. 1, pp. 1-6. EMAG'85, Newcastle.

Hunt, E. M. and Hampikian. J. M., 1997. Formation and thermal stability of aluminum nanoparticles sythesized via ion implantation into sapphire. Journal of Materials Science, accepted 30 October. 1996, in press.

Hunt, E. M., Hampikian, J. M. and Poker, D. B., 1996. Nanocrystal formation via yttrium ion implantation into sapphire. Mater. Res. Soc. Symp. Proc., 396, 403-410.

Hunt, E. M.. Hampikian, J. M. and Evans, N. D., 1996b. The examination of yttrium ion implantated alumina with energyfiltered transmission electron microscopy. In Proc. Microscopy and Microanalysis, pp. $534-535$.

Krivanek, O. L., Gubbens, A. J. and Dellby, N., 1995. Spatial resolution in EFTEM elemental maps. J. Microsc., 180, 277-287.
Reimer, L. (ed), 1995. Energy-Filtering Transmission Electron Microscopy. Springer Series in Optical Sciences. Springer-Verlag, Berlin, Heidelberg, New York.

Reimer, L., Bakenfelder, A., Fromm, I., Rennekamp, R. and Ross-Messemer, M., 1990. Electron spectroscopic imaging and diffraction. Electron Microsc. Soc. Am. Bull., 20, 73-80.

Shechtman, D., Van Heerden, D. and Josell, D., 1994. FCC titanium in Ti-Al multilayers. Mater. Lett., 205/6, 329-334.

Shuman, H., Chang, C. F. and Somlyo, A. P., 1986. Elemental imaging and resolution in energy-filtered conventional electron microscopy. Ultramicroscopy, 19, 121-134.

Wang, Z. L., 1997. Lattice imaging using plasmon energy-loss electrons, Ultramicroscopy, in press.

Wang, Z. L., 1996. Valence electron excitation and plasmon oscillations in thin films, surfaces, interfaces and small particles. Micron, 27, 265-299.

Wang, Z. L., 1991. Reflection electron imaging and spectroscopy studies of metal reduction at alpha-alumina $(0,-1,2)$ surfaces. J. Microscopy, 163, 261-274.

Wang. Z. L. and Shapiro, A. J., 1995. Energy-filtering and compositional imaging in surface and interface studies using HREM. Ultramicroscopv. 60, 115-135. 